\title{
Collaborative Research: Supporting Agency among Early Career Engineer- ing Education Faculty in Diverse Institutional Contexts
}

\section{Dr. Erin J. McCave, University of Houston}

Erin is an Instructional Assistant Professor in the Cullen College of Engineering at the University of Houston. She joined the University of Houston after completing a postdoctoral/lecturer position split between the General Engineering program and the Engineering \& Science Education Department and a $\mathrm{Ph} . D$. in Bioengineering from Clemson University. Erin's research interests include preparing students for their sophomore year, minority student engineering identity development, and providing mentoring relationships to help foster student growth and success.

\section{Dr. Courtney June Faber, University of Tennessee, Knoxville}

Courtney is a Research Assistant Professor and Lecturer in the Cook Grand Challenge Engineering Honors Program at the University of Tennessee. She completed her Ph.D. in Engineering \& Science Education at Clemson University. Prior to her Ph.D. work, she received her B.S. in Bioengineering at Clemson University and her M.S. in Biomedical Engineering at Cornell University. Courtney's research interests include epistemic cognition in the context of problem solving, and researcher identity.

\section{Dr. Cheryl A. Bodnar, Rowan University}

Cheryl A. Bodnar, Ph.D., CTDP is an Assistant Professor in the Department of Experiential Engineering Education at Rowan University. Dr. Bodnar's research interests relate to the incorporation of active learning techniques in undergraduate classes as well as integration of innovation and entrepreneurship into the engineering curriculum. In particular, she is interested in the impact that these tools can have on student perception of the classroom environment, motivation and learning outcomes. She obtained her certification as a Training and Development Professional (CTDP) from the Canadian Society for Training and Development (CSTD) in 2010, providing her with a solid background in instructional design, facilitation and evaluation. She was selected to participate in the National Academy of Engineering (NAE) Frontiers of Engineering Education Symposium in 2013 and awarded the American Society for Engineering Education Educational Research Methods Faculty Apprentice Award in 2014.

\section{Dr. Alexandra Coso Strong, Franklin W. Olin College of Engineering}

Alexandra Coso Strong is an assistant professor of systems design and engineering at Franklin W. Olin College of Engineering. Prior to starting a faculty position at Olin, she was a Postdoctoral Fellow at Georgia Tech's Center for the Enhancement of Teaching and Learning. She completed her Ph.D. in 2014 in Aerospace Engineering at Georgia Tech. Alexandra received her B.S. in Aerospace Engineering from MIT and her M.S. in Systems Engineering from the University of Virginia. Her research interests include engineering design education (especially in regards to the design of complex systems), faculty development, career pathways (both academic and industry), approaches for supporting education research-to-practice.

Dr. Walter C. Lee, Virginia Tech

Dr. Walter Lee is an assistant professor in the Department of Engineering Education and the assistant director for research in the Center for the Enhancement of Engineering Diversity (CEED), both at Virginia Tech. His research interests include co-curricular support, student success and retention, and diversity. Lee received his Ph.D in engineering education from Virginia Tech, his M.S. in industrial \& systems engineering from Virginia Tech, and his B.S. in industrial engineering from Clemson University.

\section{Dr. Courtney S. Smith-Orr, University of North Carolina, Charlotte}

Courtney S. Smith,PhD is a Teaching Assistant Professor at UNC Charlotte. Her research interests span the mentoring experiences of African American women in engineering,minority recruitment and retention, and best practices for diversity and inclusion in the Engineering classroom. 


\title{
Collaborative Research: Supporting Agency among Early Career Engineering Education Faculty in Diverse Institutional Contexts
}

\begin{abstract}
Given the infancy of engineering education as an established field and the recent increase in early career faculty aligning themselves with the discipline, it is imperative that the community better understand the experiences of these new faculty members. As a result, we will be able to enhance national efforts to train and develop faculty prepared to drive change in engineering education. Accordingly, this two-phased study investigates how institutional context influences the agency of our research team and other early career engineering education faculty as it relates to facilitating change in engineering education. Faculty play a central role in making change, thus there is a need to further understand the factors that influence their ability to do so. This work leverages collaborative inquiry and collaborative autoethnography to explore the lived experiences of our research team, which consists of six engineering education faculty who have different roles and responsibilities and are positioned in varied settings at diverse institutions. We represent a variety of perspectives with regard to our goals, visions, and training in engineering education.

This project officially started in May 2017; however, we began collecting data in August 2015. Our poster will present a summary of our current progress, which includes the use of the Q3 Research Quality workshop to guide data collection and analysis. In addition to the methodological impact of our study, the results will provide the engineering education community with evidence-based insights on conditions that facilitate change efforts by early career engineering education faculty. By sharing our findings with current and developing engineering education graduate programs, we will enable them to make programmatic changes to benefit future faculty. These findings also will provide a mechanism for divisions of the American Society of Engineering Education (ASEE) to develop programming and resources to support the sustained success of their members.
\end{abstract}

\section{Introduction and Background}

One mechanism by which the field of engineering education strives to create transformative improvements is the training and development of scholars prepared to drive change in engineering education. These individuals complete formal development programs in engineering education research in order to further the field's understanding of engineering education and to influence the engineering disciplines and educational systems. Faculty trained in engineering education research are often sought after by institutions who want to see changes within engineering curriculum and programs. As such, these faculty are in a unique position to impact change in engineering education early in their careers. Given the infancy of engineering education as a field [1] and the recent increase in both the production of $\mathrm{PhDs}$ in engineering education and faculty members aligning themselves with the discipline, it is imperative that we develop a better understanding of the experiences of these community members.

To achieve impact, early career engineering education faculty need to be involved in "taking strategic and intentional actions or perspectives towards goals that matter to oneself,” [2, p. 50], [3]. In other words, they need to exercise agency. Impact in engineering education can occur in a variety of ways, whether through the design of innovative curricula, development of educational 
policy initiatives, creation of supportive learning environments, translation of engineering education research into practice, or conversations with students about difficult content and career paths. However, despite the progress made in the past, there are still many challenges to overcome, particularly if trying to create large-scale transformations [4].

Accordingly, the aim of this work is to investigate how institutional context influences the agency of early career engineering education faculty as it relates to facilitating change. To do so, we are systematically examining our own experiences as six early career faculty members in diverse settings. Subsequently, we will examine the experiences of 12-15 additional early career faculty members. Through this process, we will (1) advance the engineering education community's understanding of existing structures for facilitating change (or the lack thereof) in engineering education; (2) identify barriers and supports for making change as early career engineering education faculty; and (3) develop a co-constructed understanding of how to better prepare and support faculty to exercise agency as it relates to impacting engineering education during their first few years in academic positions.

\section{Executive Summary}

Project Outcomes and Significance

We will reach our project aims by specifically addressing the following qualitative research questions:

RQ1. What impact do early career faculty members hope to have within engineering education?

RQ2. How do (a) institutional, (b) individual, and (c) disciplinary field and societal features influence early career engineering education faculty member's agency to impact engineering education in their particular positions?

RQ3. How do early career faculty members perceive their impact on engineering education at their institution and more broadly?

We are answering these questions through a two-phase qualitative study. Phase I leverages both collaborative inquiry and collaborative autoethnography, guiding our exploration of our lived experiences and respective academic cultures. Initially focusing on our own experiences, as early career engineering education faculty, allows a deeper understanding of our experiences, both good and bad, that may not be revealed in a less intimate approach. The longitudinal nature of our approach also makes it possible for us to document and reflect on our experiences and how we navigate obstacles. Phase II will use constant comparative methods to expand and refine Phase I findings through a series of semi-structured interviews with 12-15 additional pre-tenure engineering education faculty.

This study will produce the following outcomes:

Outcome 1: A conceptual model that depicts the relationship between institutional context and faculty agency among early career engineering education faculty Outcome 2: Best practices for establishing a cross-institutional community of practice to support early career engineering education faculty

Outcome 3: Key recommendations for better supporting engineering education PhDs and early career faculty aligned with the discipline 
Outcome 4: A methodological foundation that can be used to empirically investigate and better understand institution-specific problems that require a more intimate data collection and analysis process

Research Methods

Our multiphase, longitudinal research approach is allowing us to deeply and systematically explore our own experiences and the transferability of our results to the larger engineering education community. Figure 1 provides an overview of our project.

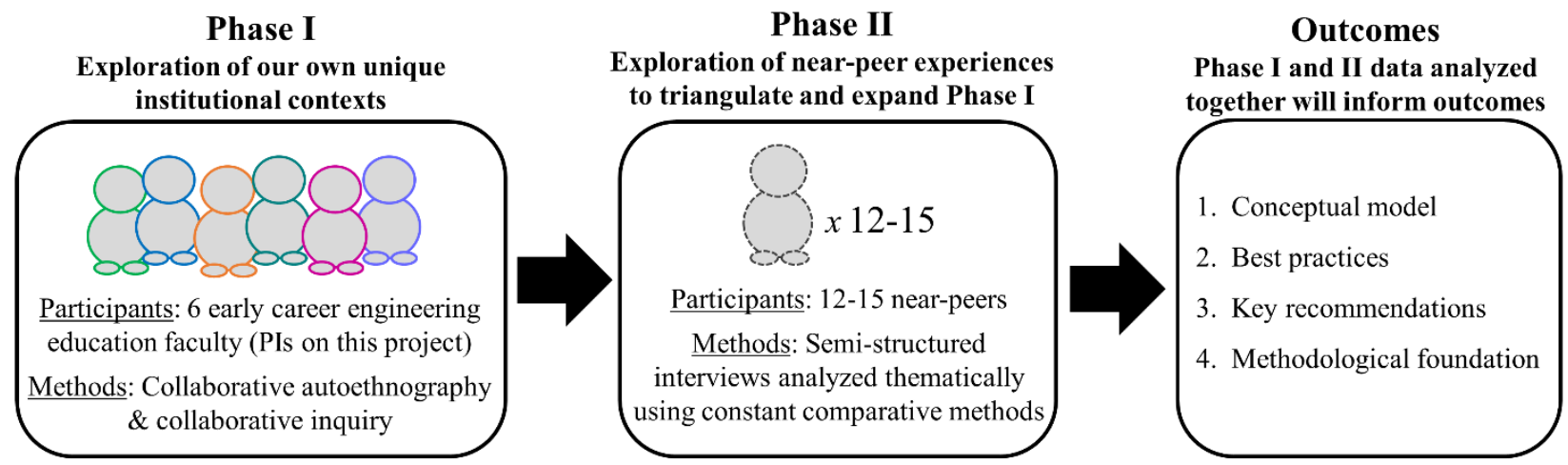

Figure 1: Overview of project phases and outcomes.

In Phase I, we are using collaborative inquiry and collaborative autoethnography methods. Combined, these methods are guiding our use of iterative cycles of reflection and action as we explore academic cultures. We are documenting our experiences through weekly reflections, group discussions of relevant literature, and virtual meetings. During these meetings, we are discussing our experiences and seeking advice about our current challenges, ultimately leading to changes in our practices. Through our experiences, we seek to develop a preliminary conceptual model that depicts the relationship between individual, organizational, and societal supports and barriers to our agency as engineering education faculty members. This preliminary conceptual model will depict the relationship between institutional context and faculty agency, highlighting influences from the individual, organization, and field level.

In Phase II, we will use a constant comparative approach to refine our preliminary model. We will conduct a series of semi-structured interviews with 12-15 pre-tenure engineering education faculty members who work in a diverse set of institutional contexts, allowing us to validate and expand the findings from Phase I. These interviews and the subsequent data analysis will be conducted alongside engineering education graduate students.

Project Status and Future Work

This project officially started in May 2017; however, we have been collecting data since August 2015. Our poster will present a summary of our current progress, which includes the use of the Q3 framework and a Q3 Research Quality workshop to guide our plans for data collection and analysis. Leveraging the Q3 framework [5] and an in-person workshop was advantageous to systematically examine how we would combine elements from collaborative autoethnography and collaborative inquiry, which are traditionally not combined, to explore our research questions from an interpretive perspective. An example of how we have been able to leverage the 
Q3 framework and Q3 Research Quality workshop can be found in our ASEE paper published this year documenting the development of an analysis framework [6].

Along with the continued development and documentation of our methodology, we have been exploring two particular aspects of our experiences: our transition into new faculty positions through our perceptions of our experiences during our first two years within these roles and our perceptions of our own impact on engineering education. Using Schlossberg's Transition Theory [7] as a guiding framework, we each created critical incident timelines of our experiences in an effort to isolate key supports, barriers, and strategies we experienced and used to aid our transition into our individual faculty positions [6]. Transition theory, created to describe transitions within adult development, has been expanded to explore contexts such as pathways into and through different experiences. This provided us a framework to explore how our roles, routines, and professional relationships change as we transitioned into our diverse faculty positions. The results of this exploration will allow us to not only better understand our own experiences and the experiences of our colleagues, but also begin to isolate factors affecting our agency and how these can be addressed to improve the agency of future early career engineering educators.

To explore the connection between agency and impact, we also need to examine our perceptions of our own impact on engineering education. What areas do we want to have impact in? To what extent has that impact been achieved? Why or why not? Leveraging London's [8] Scientific, Contextual, and Societal Impact Framework, we completed two impact-focused written reflections and participated in a mini-impact workshop to help identify the different areas we have already had impact in, as well as, those we want to pursue over the next few years of our career. We will be using these reflections to determine if there are any similarities and differences between the scientific, contextual and societal impacts that we can achieve based on our institutional contexts and differences in positions. These activities will serve as a foundation for the development of the preliminary model, which will guide our work in Phase II.

In addition to our study's methodological impact, the results will provide the engineering education community with evidence-based insights on conditions that facilitate change efforts by early career engineering education faculty. By sharing our findings with current and developing engineering education graduate programs, we will enable them to make programmatic changes to benefit future faculty. These findings also provide a mechanism for divisions within ASEE to develop programming and resources to support the sustained success of their members.

\section{Acknowledgements}

This material is based upon work supported by the National Science Foundation under Grant Numbers 1663909, 1664217, 1664038, 1664016, 1664008, 1738262. Any opinions, findings, and conclusions or recommendations expressed in this material are those of the author(s) and do not necessarily reflect the views of the National Science Foundation.

\section{References}

[1] J. Froyd and J. R. Lohmann, "Chronological and Ontological Development of Engineering Education as a Field of Scientific Inquiry,” in Cambridge Handbook of Engineering Education Research, A. Johri and B. Olds, Eds. Cambridge University Press, 2014, pp. 328. 
[2] C. M. Campbell and K. A. O’Meara, “Faculty Agency: Departmental Contexts that Matter in Faculty Careers,” Res. High. Educ., vol. 55, no. 1, pp. 49-74, 2014.

[3] K. A. O’Meara, C. M. Campbell, and A. Terosky, "Living Agency in the Academy: A Conceptual Framework for Research and Action,” in Annual Meeting of the Association for the Study of Higher Education, 2011.

[4] A. F. McKenna, J. Froyd, and T. Litzinger, "The complexities of transforming engineering higher education: Preparing for next steps,” J. Eng. Educ., vol. 103, no. 2, pp. 188-192, 2014.

[5] J. Walther, N. Sochacka, and N. Kellam, "Research Quality Workshop,” in Frontiers in Education Conference, 2014.

[6] A. Coso Strong, C. Smith-Orr, C. Bodnar, W. Lee, C. Faber, and E. McCave, "Using a Critical Incident-Centered Transition Theory Framework to Explore Engineering Education Faculty Transitions,” in American Society for Engineering Education Annual Conference, 2018.

[7] N. K. Schlossberg, E. B. Waters, and J. Goodman, Counseling adults in transition: Linking practice with theory, 2nd ed. New York: Springer Publishing Company, 1995.

[8] J. London, “A Content Analysis of How STEM Education Researchers Discuss the Impact of their Publicly-Supported Research.,” Int. J. Eng. Educ., 2018. 